Article

\title{
In Vitro Effects of Sulforaphane on Interferon-Driven Inflammation and Exploratory Evaluation in Two Healthy Volunteers
}

\author{
Elena Genova ${ }^{1,+}\left(\mathbb{D}\right.$, Maura Apollonio ${ }^{1,+}\left(\mathbb{D}\right.$, Giuliana Decorti ${ }^{1,2} \mathbb{D}$, Alessandra Tesser ${ }^{1, *} \mathbb{C}$, \\ Alberto Tommasini ${ }^{1,2, \ddagger(D)}$ and Gabriele Stocco ${ }^{3, \ddagger}$ \\ 1 Institute for Maternal and Child Health IRCCS Burlo Garofolo, 34137 Trieste, Italy; \\ elena.genova@burlo.trieste.it (E.G.); maura.apollonio@burlo.trieste.it (M.A.); \\ decorti@units.it (G.D.); alberto.tommasini@burlo.trieste.it (A.T.) \\ 2 Department of Medical, Surgical and Health Sciences, University of Trieste, 34149 Trieste, Italy \\ 3 Department of Life Sciences, University of Trieste, 34127 Trieste, Italy; stoccog@units.it \\ * Correspondence: alessandra.tesser@burlo.trieste.it; Tel.: +39-040-378-5422 \\ + These authors contributed equally to this work. \\ $\ddagger$ These authors contributed equally to this work.
}

\section{check for} updates

Citation: Genova, E.; Apollonio, M.; Decorti, G.; Tesser, A.; Tommasini, A.; Stocco, G. In Vitro Effects of Sulforaphane on Interferon-Driven Inflammation and Exploratory Evaluation in Two Healthy Volunteers. Molecules 2021, 26, 3602. https: / / doi.org/10.3390/ molecules 26123602

Academic Editors: Sokcheon Pak and Soo Liang Ooi

Received: 14 April 2021

Accepted: 10 June 2021

Published: 12 June 2021

Publisher's Note: MDPI stays neutral with regard to jurisdictional claims in published maps and institutional affiliations.

Copyright: (c) 2021 by the authors. Licensee MDPI, Basel, Switzerland. This article is an open access article distributed under the terms and conditions of the Creative Commons Attribution (CC BY) license (https:// creativecommons.org/licenses/by/ $4.0 /)$.

\begin{abstract}
Interferonopathies are rare genetic conditions defined by systemic inflammatory episodes caused by innate immune system activation in the absence of pathogens. Currently, no targeted drugs are authorized for clinical use in these diseases. In this work, we studied the contribution of sulforaphane (SFN), a cruciferous-derived bioactive molecule, in the modulation of interferon-driven inflammation in an immortalized human hepatocytes (IHH) line and in two healthy volunteers, focusing on STING, a key-component player in interferon pathway, interferon signature modulation, and GSTM1 expression and genotype, which contributes to SFN metabolism and excretion. In vitro, SFN exposure reduced STING expression as well as interferon signature in the presence of the pro-inflammatory stimulus cGAMP (cGAMP $3 \mathrm{~h}$ vs. SFN+cGAMP $3 \mathrm{~h} p$ value <0.0001; cGAMP $6 \mathrm{~h}$ vs. SFN+cGAMP $6 \mathrm{~h} p<0.001$, one way ANOVA), restoring STING expression to the level of unstimulated cells. In preliminary experiments on healthy volunteers, no appreciable variations in interferon signature were identified after SFN assumption, while only in one of them, presenting the GSTM1 wild type genotype related to reduced SFN excretion, could a downregulation of STING be recorded. This study confirmed that SFN inhibits STING-mediated inflammation and interferonstimulated genes expression in vitro. However, only a trend towards the downregulation of STING could be reproduced in vivo. Results obtained have to be confirmed in a larger group of healthy individuals and in patients with type I interferonopathies to define if the assumption of SFN could be useful as supportive therapy.
\end{abstract}

Keywords: sulforaphane; type I interferons; STING; interferon signature; GSTM1

\section{Introduction}

The mechanisms behind inflammatory diseases are complex and various, making it difficult to find targeted therapies for patients. From the second part of the last century, it was clear that glucocorticoids could represent an effective anti-inflammatory treatment for the vast majority of inflammatory diseases, although burdened by serious adverse effects [1]. However, at the end of the last century, a better knowledge of the pathogenetic mechanisms of these diseases allowed for the development of targeted therapies able to mimic the anti-inflammatory power of cortisone without its adverse effects. For example, following the discovery that some diseases are dominated by the inflammatory effect of specific cytokines, biological drugs usually based on monoclonal antibodies, soluble receptors, or receptor antagonists have been developed [2]. Indeed, anti-tumor necrosis 
factor- $\alpha(\mathrm{TNF} \alpha)$ antibodies have been successfully used in rheumatoid arthritis and in inflammatory bowel disease [3,4], while interleukin-1 (IL-1) inhibitors have found application in autoinflammatory diseases, such as Still's disease, recurrent pericarditis, gout arthritis, Behçet's disease, and a set of genetic conditions such as periodic fever [5]. In the last ten years, a set of genetic diseases characterized by a defective regulation of type I interferons production, the so-called interferonopathies, were identified, which often showed poor responses to conventional anti-inflammatory drugs, including biologics and glucocorticoids. Thus, their study allowed to evaluate the potential of novel targeted treatments to reduce interferon driven inflammation [6,7]. Interferonopathies are a heterogeneous group of Mendelian diseases characterized by an abnormal response to nucleic acid stimuli due to either deficiency of nucleases involved in the disposal of nucleic acids, or to defective regulation of downstream effector molecules, leading to excessive production of type I interferon, in particular $\alpha$ and $\beta[6,7]$. Interferons are glycoproteic cytokines classified in type I, II, and III according to their cellular origin and receptor structure. Interferons can activate several transduction pathways by different mechanisms resulting in antiviral, immunomodulatory and antiproliferative activities $[8,9]$. Interferonopathies include Aicardi-Goutières syndrome (AGS), monogenic forms of systemic lupus erythematosus (SLE), STING-associated vasculopathy with onset in infancy (SAVI), COPA syndrome and other exceptionally rare disorders [6]. Unfortunately, the measure of type I interferon in human sera is not routinely available in clinical practice, due to the short half-life and the low serum concentrations of the cytokine. Moreover, an isolated interferon dosage may not fully reflect the importance of a prolonged systemic exposure. These issues restrict the ability to diagnose and monitor treatment of these diseases [10]. So far, the assessment of interferon-mediated inflammation in these disorders relies on indirect assays, performed on peripheral blood cells, that present transcriptional changes related to their autocrine or paracrine exposition to high concentration of interferons [11]. One of these approaches consists in the relative quantification of a set of interferon-stimulated genes (ISGs), the so-called interferon signature. One of the most used set of ISGs for interferon signature assessment was proposed by Crow and his group [10], who defined the over-expression of six ISGs (IFI27, IFI44L, IFIT1, ISG15, RSAD2, and SIGLEC1) in a cohort of AGS patients compared to healthy controls. The interferon signature intensity is provided by the calculation of an "interferon score" (IFN score) as the median fold change of the six target ISGs. The validation of this score for the detection of monogenic interferonopathies made it preferred by centers involved in the screening and diagnosis of these rare conditions.

Currently, no targeted drugs for type I interferonopathies are authorized for routine clinical use and the few treatments available control principally the downstream effects of interferons [6,7]. Only a few drugs, such as antimalarial agents, Janus Kinase inhibitors, mycophenolate mofetil, and high dose glucocorticoids, have proven to demonstrate some efficacy. Moreover, anti-interferon $\alpha$ antibodies resulted not useful in the clinical practice while, more recently, antibodies blocking the common type I interferon receptor have been developed and are used with promising results in SLE [12]. To identify more efficient targeted drugs, and for developing drug classes already proven as partially efficient, it is important to develop or improve in vitro models already available reproducing significant disease-related pathogenic mechanisms $[13,14]$. During the research of new effective treatments, it is also crucial to face the possibility of the infectious risk connected to excessive suppression of cytokines and organism signaling pathways [15]. This implies that active compounds interacting with these inflammatory mechanisms should be modular in action intensity and should be selective, in order to reduce adverse effects.

Historically, medicinal chemistry finds a landmark in plants as a starting point for drug development. In the last years, the field of nutraceuticals has expanded, providing treatments that maintain the bioactive plant compound as close to its native state as possible $[16,17]$. In this context, sulforaphane (SFN), a bioactive molecule contained in cruciferous vegetables (e.g., broccoli), emerges as a potential phytochemical compound, able to produce positive results in conditions lacking satisfactory pharmaceutical compounds [18], 
by modulating an important key-component of interferon pathway production. Despite being a food-derived molecule, SFN can reach an intracellular concentration sufficient to affect gene expression, thanks to its high bioavailability [17]. In particular, based on the in vitro effect on cells from patients with SAVI syndrome, SFN may be a reasonable supplement in the treatment of patients affected by type I interferonopathies. Indeed, it is well-known that SFN is an effective activator of the transcription factor NRF2 (nuclear factor erythroid 2-related factor 2), which can modulate key components of the cellular defense processes, operating on redox- and inflammation-regulating gene expression via activation of the antioxidant responsive elements axis [19]. Cytosolic NRF2 contrasts the activity of nuclear factor NF- $\mathrm{kB}$, which drives immune responses to cellular challenges such as bacterial and viral infection and inflammation [19]. As regards the interferon cascade, NRF2 activation driven by SFN leads to downregulation of STING (stimulator of interferon genes), an important kinase implicated in type I interferon production, through a mechanism that brings to its mRNA instability in a time and dose dependent manner [20]. In addition, SFN has been demonstrated as an important agent in the regulation of functionalizing (phase I) and conjugating (phase II) xenobiotic biotransformation enzymes [21]. Among phase II enzymes, GSTs are known to be induced by SFN through the activation of the antioxidant responsive elements axis thanks its sulphur interaction with thiol groups of the Keap1 cysteine residues [22]. In addition, GSTs, and in particular the GSTM1 isoform, play an important role in enzymatic formation and cleavage of the GSH conjugates of isothiocyanates, contributing to SFN pharmacokinetics [23-25]. In fact, higher SFN excretion in urines after $24 \mathrm{~h}$ since consumption in null individuals rather than those with functional GSTM1 was identified [25]. This evidence suggested that GSTM1 positive individuals may have a different metabolism of SFN, with reduced SFN metabolites excretion. This may explain why GSTM1 null individuals show less protection offered by SFN than positive subjects do [25].

In this work we studied the effect of SFN on interferon inflammation induced by cGAMP treatment, using a healthy immortalized human hepatocytes (IHH) cell line, focusing on STING and interferon signature modulation. The peculiar ability of SFN to induce the expression of phase II enzyme GSTM1, that plays an important role in its pharmacokinetics, was also evaluated in IHH cells. Moreover, as a secondary objective, we assessed the expression of STING and interferon signature in vivo in two healthy volunteers after the consumption of increasing doses of two commercial SFN supplements.

\section{Results}

\subsection{Cytotoxicity of SFN on IHH Cells}

To evaluate cytotoxicity of SFN on IHH cells, various concentrations were tested $\left(1.25 \times 10^{-6} \mathrm{M}\right.$ to $\left.4 \times 10^{-5} \mathrm{M}\right)$ for $72 \mathrm{~h}$ by MTT assay. IHH cell line was found sensitive to SFN (EC $501.84 \times 10^{-5} \mathrm{M}$, confidence intervals C.I. $1.37 \times 10^{-5} \mathrm{M}$ to $2.49 \times 10^{-5} \mathrm{M}$ ) (Figure 1). The $10 \mu \mathrm{M}$ concentration used for the subsequent treatment resulted in about $70 \%$ of cell viability.

\subsection{STING Expression}

STING expression in IHH cells was evaluated by RT-PCR. Cells were treated with $10 \mu \mathrm{M}$ SFN in the presence or absence of the inflammatory stimulus $5.9 \mu \mathrm{M}$ cGAMP added in the last 3 or $6 \mathrm{~h}$ of SFN incubation (Figure 2). 


\section{SFN treatment}

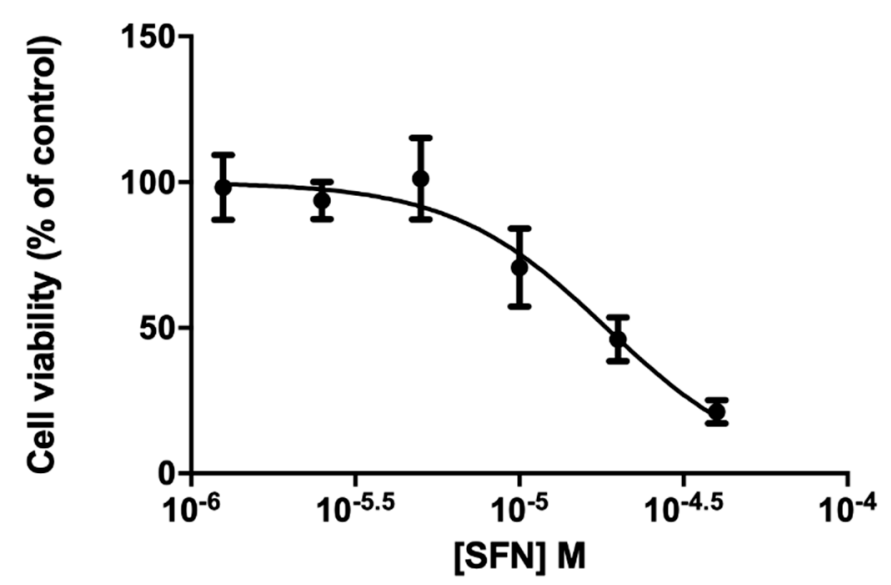

Figure 1. Cytotoxicity effects of sulforaphane (SFN) on IHH cell line. Cells were exposed for $72 \mathrm{~h}$ to SFN and cytotoxicity effects were analyzed by MTT assay. Data are reported as means \pm SE of 3 independent experiments performed in triplicate. O.D.\% observed to untreated cells.

$\mathrm{IHH}$

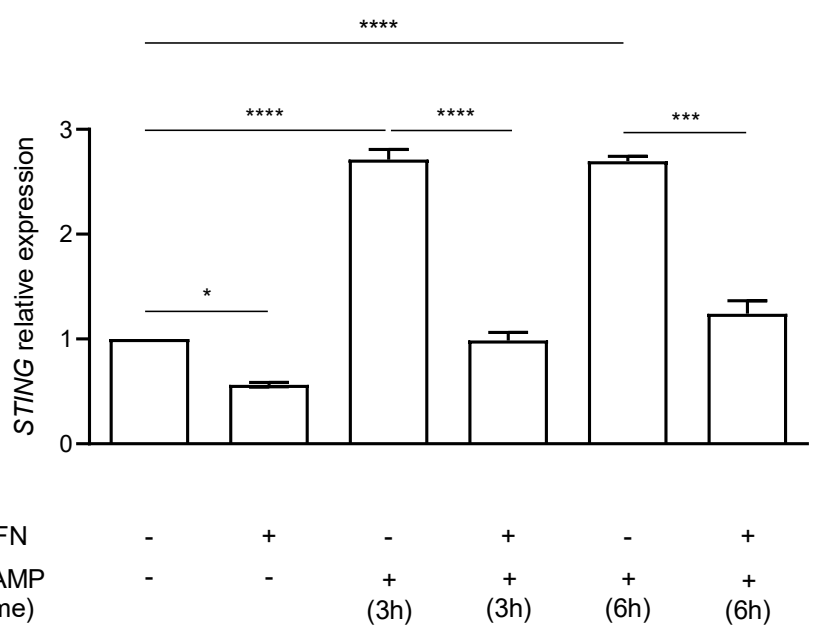

Figure 2. STING expression in IHH cells pretreated or not with sulforaphane (SFN, $10 \mu \mathrm{M})$ in presence of inflammatory stimulus cGAMP $(5.9 \mu \mathrm{M})$. Data are shown as means and C.I. of two representative experiments and reported evaluating $2^{-\Delta \Delta \mathrm{Ct}}$ values using untreated cells as calibrator and HPRT1 and G6DP housekeeping genes as reference. *: $p<0.05$, one way ANOVA untreated CTRL IHH cells vs. $72 \mathrm{~h}$ SFN $10 \mu \mathrm{M}$ treatment. ${ }^{* * *}: p<0.0001$, one way ANOVA IHH exposed to $5.9 \mu \mathrm{M}$ cGAMP for $3 \mathrm{~h}$ and vs. IHH pre-treated with SFN $10 \mu \mathrm{M}$ for $72 \mathrm{~h}$ and $5.9 \mu \mathrm{M}$ cGAMP for $3 \mathrm{~h}$. ***: $p<0.001$, one way ANOVA IHH exposed to $5.9 \mu \mathrm{M}$ cGAMP for $6 \mathrm{~h}$ vs. IHH pre-treated with SFN $10 \mu \mathrm{M}$ for $72 \mathrm{~h}$ and $5.9 \mu \mathrm{M}$ cGAMP for $6 \mathrm{~h} .{ }^{* * *}: p<0.0001$, one way ANOVA untreated CTRL IHH cells vs. IHH treated with $5.9 \mu \mathrm{M}$ cGAMP for 3 and $6 \mathrm{~h}$.

As expected, cGAMP induced a strong increase $(p<0.0001$, one way ANOVA) in STING expression after 3 and 6 h of stimulation. A significant ( $p<0.05$, one way ANOVA) STING expression decrease was identified in cells treated with SFN for $72 \mathrm{~h}$ in comparison to the untreated control (CTRL) (Figure 2). The effect was even more evident when STING expression was induced after $3 \mathrm{~h}$ and $6 \mathrm{~h}$ of cell exposure to the inflammatory stimulus cGAMP in comparison to control. Cells pre-treated with SFN and stimulated with cGAMP showed a significant lower STING expression in comparison to those that were only stimulated with cGAMP (cGAMP $3 \mathrm{~h}$ vs. SFN+cGAMP $3 \mathrm{~h} p$ value $<0.0001$; cGAMP $6 \mathrm{~h}$ vs. SFN+cGAMP $6 \mathrm{~h} p<0.001$, one way ANOVA) resulting in STING levels similar to the untreated control. 


\subsection{Interferon Signature Analysis of IHH Cells}

Interferon signature was analyzed on IHH cells treated with SFN $10 \mu \mathrm{M}$ in presence and absence of the inflammatory stimulus cGAMP, to evaluate whether the expression of the six ISGs decreases in the presence of SFN during an inflammatory event (Figure 3).

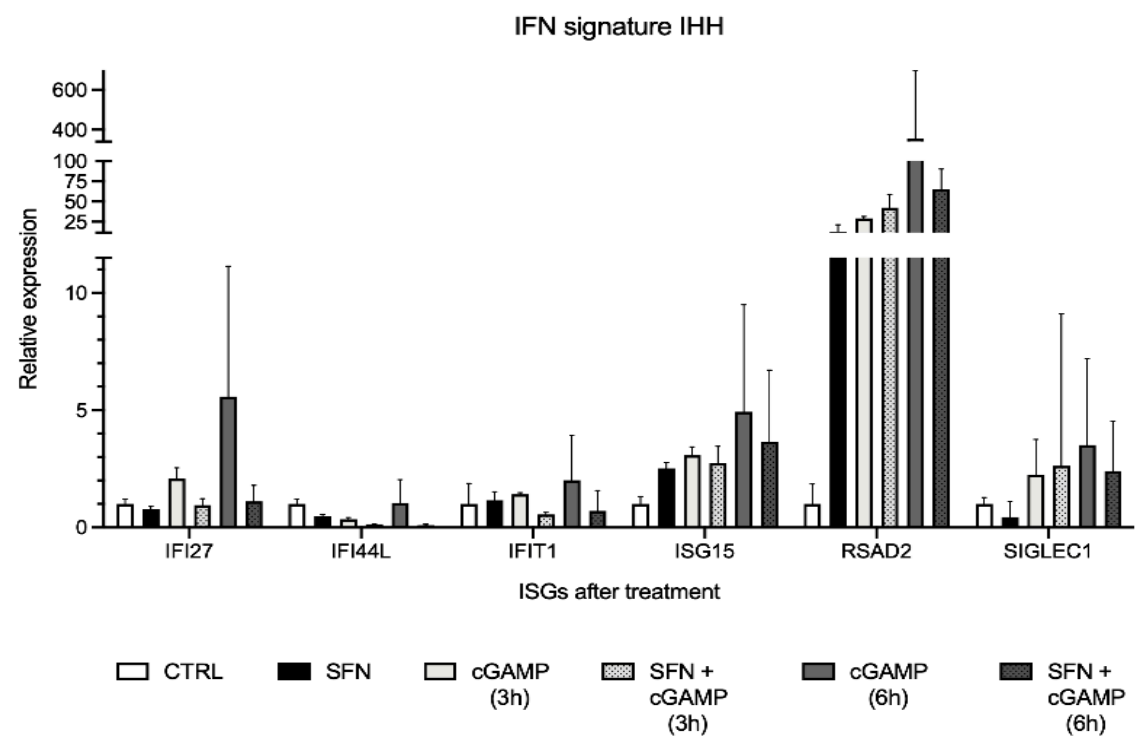

Figure 3. Interferon signature analysis of IHH cells pretreated with sulforaphane (SFN, $10 \mu \mathrm{M})$ for $72 \mathrm{~h}$ in presence of inflammatory stimulus cGAMP $(5.9 \mu \mathrm{M})$ displayed as the expression levels of the six interferon-stimulated genes (ISGs). Data are shown as means and C.I. of one representative experiment and reported evaluating $2^{-\Delta \Delta \mathrm{Ct}}$ values using untreated cells as calibrator (CTRL) and HPRT1 and G6DP housekeeping genes as reference.

An increase of ISGs expression after cell exposure to the cGAMP inflammatory stimulus was identified (Figure 3). In particular, the increment was higher after $6 \mathrm{~h}$ exposure in comparison to $3 \mathrm{~h}$. Most ISGs showed a lower expression level in cells pretreated with SFN and subsequently exposed to cGAMP. In particular, the effect was more evident when cells were pretreated with SFN for $72 \mathrm{~h}$ and stimulated with cGAMP for $6 \mathrm{~h}$ in comparison to the same conditions at $3 \mathrm{~h}$. Above all ISGs, RSAD2 proved the most represented and overexpressed gene during cell stimulation with cGAMP and pretreatment with SFN for $72 \mathrm{~h}$ significantly reduced its overexpression. However, we noticed a strong increment in RSAD2 expression also after SFN exposure alone, in absence of the cGAMP stimulus. Also, ISG15 expression was slightly augmented by SFN exposure. The intensity of the interferon signature analyses is reported in Table 1 as IFN scores.

Table 1. IFN scores of IHH cells treated with SFN and cGAMP.

\begin{tabular}{cccccc}
\hline CTRL & SFN 72 h & cGAMP 3 h & $\begin{array}{c}\text { SFN 72 h + } \\
\text { cGAMP 3 h }\end{array}$ & cGAMP 6 h & $\begin{array}{c}\text { SFN 72 h + } \\
\text { cGAMP 6 h }\end{array}$ \\
\hline 1.00 & 0.96 & 2.16 & 1.79 & 4.22 & 1.75 \\
\hline
\end{tabular}

$\overline{\mathrm{CTRL}}=$ untreated control; SFN = sulforaphane $10 \mu \mathrm{M} ; \mathrm{cGAMP}=5.9 \mu \mathrm{M}$.

IFN score (Table 1) was higher in cells stimulated with the proinflammatory stimulus cGAMP. In particular, IFN score resulted about two times higher when the stimulus was maintained for a longer time. By contrast, the IFN score was similar, considerably lower in cells pre-treated with SFN and then stimulated with cGAMP for 3 or $6 \mathrm{~h}$. Cells treated only with SFN have an IFN score similar to the control. 


\subsection{IHH GSTM1 Analysis}

GSTM1 expression was evaluated by RT-PCR after SFN exposure for 24 and $72 \mathrm{~h}$ at a concentration of $10 \mu \mathrm{M}$ (Figure 4).

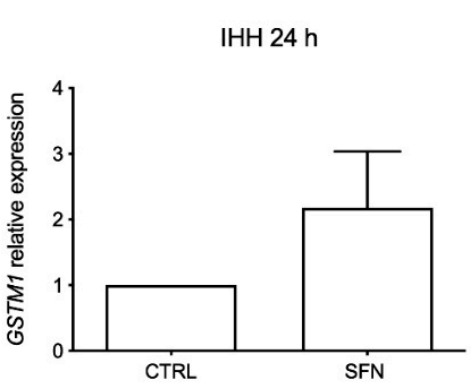

(a)

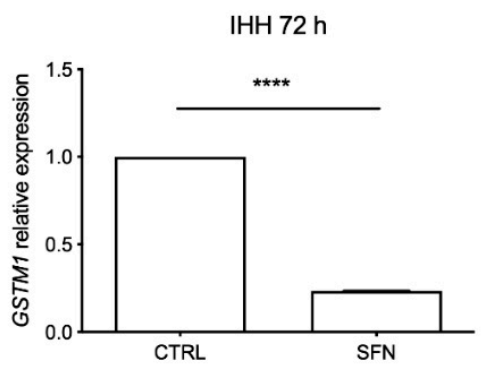

(b)

Figure 4. GSTM1 expression in IHH cell line treated with sulforaphane (SFN, $10 \mu \mathrm{M})$ for $24 \mathrm{~h}$ (a) and $72 \mathrm{~h}(\mathbf{b})$. Data are shown as means and C.I. of three representative experiments and reported evaluating $2^{-\Delta \Delta \mathrm{Ct}}$ values, using untreated cells as calibrator (CTRL) and beta-actin housekeeping gene as reference. ${ }^{* * *}: p<0.001$, one way ANOVA IHH exposed to SFN vs. untreated CTRL IHH cells.

GSTM1 expression increased after $24 \mathrm{~h}$ of treatment while significantly decreased after $72 \mathrm{~h}$ of SFN incubation ( $p<0.001$, one way ANOVA).

\subsection{Basal STING Expression in Healthy Volunteers and Patients}

Initially, basal STING expression in six patients suffering from type I interferonrelated disorders (SLE, AGS, CANDLE like) and in three healthy individuals, not taking drugs or supplements, was evaluated to identify possible differences between healthy individuals and patients. The expression reported is normalized to a control (CTRL), which is represented by one of the three healthy individuals (Figure 5).

\section{PATIENTS AND HEALTY VOLUNTEERS}

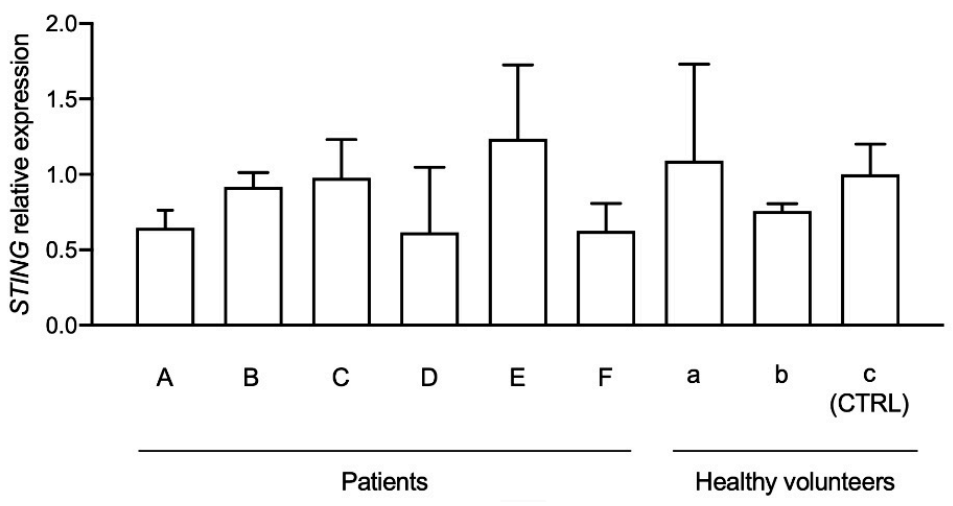

Figure 5. STING expression in patients (A-F) suffering from type I interferon dysregulations and healthy individuals $(\mathrm{a}-\mathrm{c})$. Data are reported evaluating $2^{-\Delta \Delta \mathrm{Ct}}$ values, using healthy volunteer " $\mathrm{c}^{\text {" }}$ as calibrator (CTRL) and HPRT1 and G6DP housekeeping genes as reference.

Results indicated that STING expression in patients and healthy volunteers is heterogenous and no relevant differences between healthy individuals and patients can be observed. 


\subsection{STING Expression after Administration of Lower-Dose SFN Supplement in HV1}

HV1 was treated with up to $25.2 \mathrm{mg}$ of SFN daily for three consecutive days (T1: $24 \mathrm{~h}$ of treatment, T2: $72 \mathrm{~h}$ of treatment) and, after two weeks of interruption, for other seven days (T3) to evaluate whether oral SFN administration can decrease STING expression. The relative expression was evaluated considering STING expression measured in the last day of supplement intake as reference (T3) (Figure 6).

\section{HV1, LOWER DOSE}

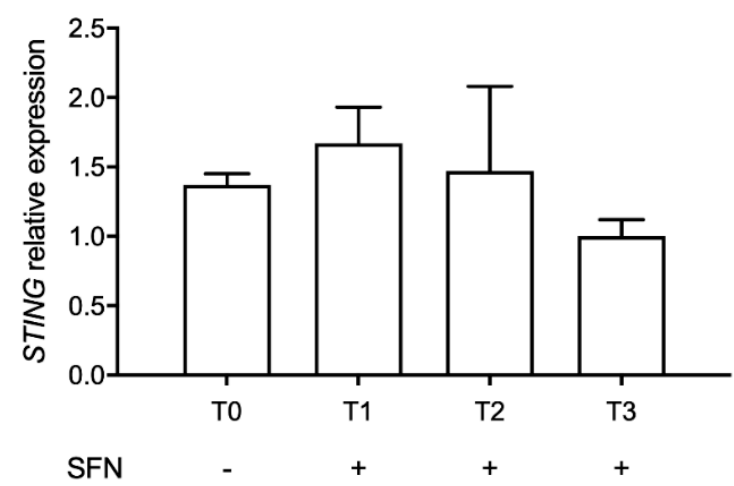

Figure 6. STING expression after sulforaphane (SFN) supplement consumption in the first volunteer (HV1). Data are reported evaluating $2^{-\Delta \Delta C t}$ values considering the last day of assumption as calibrator (T3), using HPRT1 and G6DP housekeeping genes as reference.

Results showed no significant changes in STING expression.

\subsection{Interferon Signature Analysis after Administration of Lower-Dose SFN Supplement in HV1}

To evaluate whether interferon signature analysis could be affected by oral SFN supplement administration, the expression levels of the six ISGs were assessed on the same samples used for STING analysis of the same volunteer (HV1), considering as calibrator a pool of healthy volunteers' cDNAs (CTRL) (Figure 7).

IFN signature HV1, LOWER DOSE

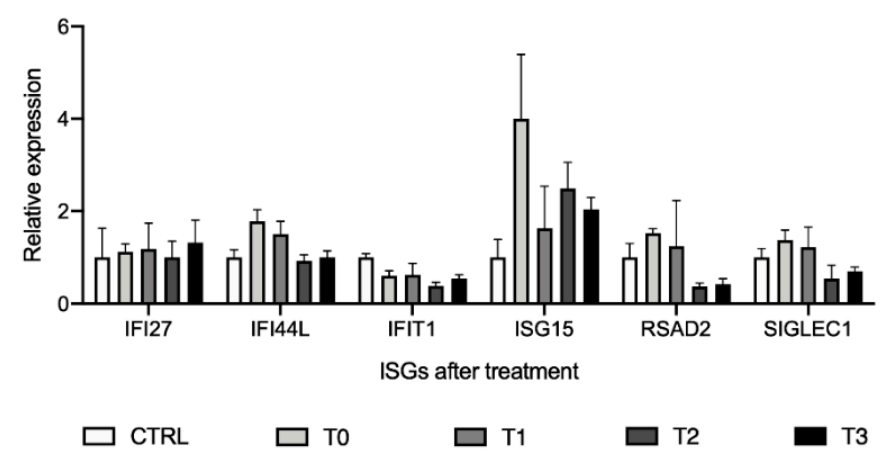

Figure 7. Interferon signature analysis after sulforaphane (SFN) supplement assumption in the first volunteer (HV1) displayed as the expression levels of the six interferon-stimulated genes (ISGs). Data are shown as means and C.I. of one representative experiment reported evaluating $2^{-\Delta \Delta \mathrm{Ct}}$ values considering a pool of healthy volunteers' cDNAs as calibrator and using HPRT1 and G6DP housekeeping genes as reference.

Relative gene expression showed a reduction of the expression levels of most of the ISGs after SFN assumption, even if not significant. The intensities of interferon signature analyses, reported as IFN scores (Table 2), did not show significant changes for different intake times. 
Table 2. IFN scores calculated through the median of the relative quantifications of the six genes of the interferon signature analysis after administration of lower-dose sulforaphane (SFN) supplement in the first healthy volunteer (HV1).

\begin{tabular}{ccccc}
\hline CTRL & T0 & T1 & T2 & T3 \\
\hline 1.00 & 1.44 & 1.23 & 0.73 & 0.85 \\
\hline
\end{tabular}

2.8. STING Expression after Higher-Dose SFN Supplement Administration in the Two Volunteers

After gene expression evaluation in HV1 taking lower supplement doses, we enrolled a second volunteer (HV2), increased the SFN dose and changed the commercial supplement (Broccoraphan ${ }^{\circledR}$, Dieters). The dose was about four folds higher ( $90 \mathrm{mg} /$ day) in comparison to the first one (up to $25.2 \mathrm{mg} /$ day), and administration time lasted for three days (T1, T2, T3). Relative STING expression was evaluated before (T0) and after SFN assumption, considering as control the last day of supplement consumption (T3) (Figure 8).

HV1, HIGHER DOSE

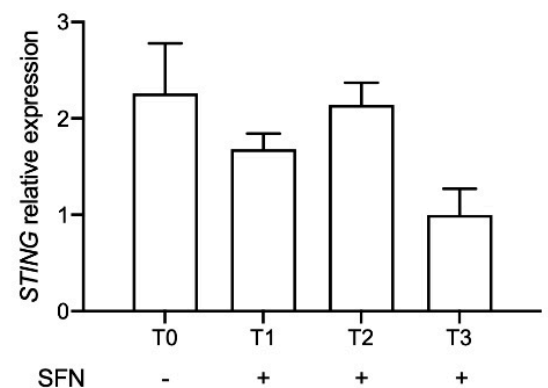

HV2, HIGHER DOSE

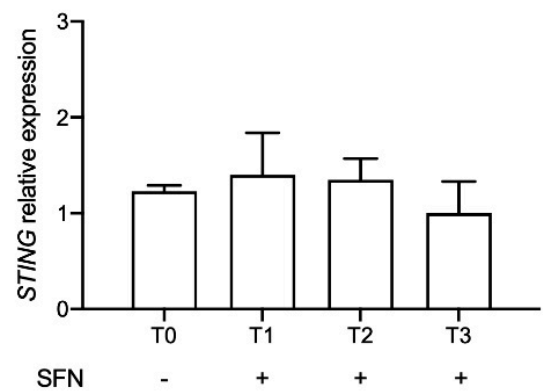

Figure 8. STING expression in two healthy volunteers (HV1, HV2) after sulforaphane (SFN) supplement administration at $90 \mathrm{mg} /$ die for three days. Data are reported evaluating $2^{-\Delta \Delta \mathrm{Ct}}$ values considering the last day of supplement consumption (T3) as calibrator and using HPRT1 and G6DP housekeeping genes as reference.

A downward trend in STING expression was observed in HV1 but not in HV2.

2.9. Interferon Signature Analysis after Higher-Dose SFN Supplement Assumption in the Two Volunteers

To evaluate the ISGs expression levels after consumption of a higher dose of SFN supplement (90 mg/day), interferon signature analysis was performed before (T0) and after SFN assumption (T1, T2, T3). ISGs expression results are relative to the calibrator, which is represented by a pool of healthy volunteers' cDNAs (CTRL). Results did not highlight significant changes in ISGs after the $90 \mathrm{mg} /$ day higher dose of SFN supplement in both volunteers (Figure 9). 


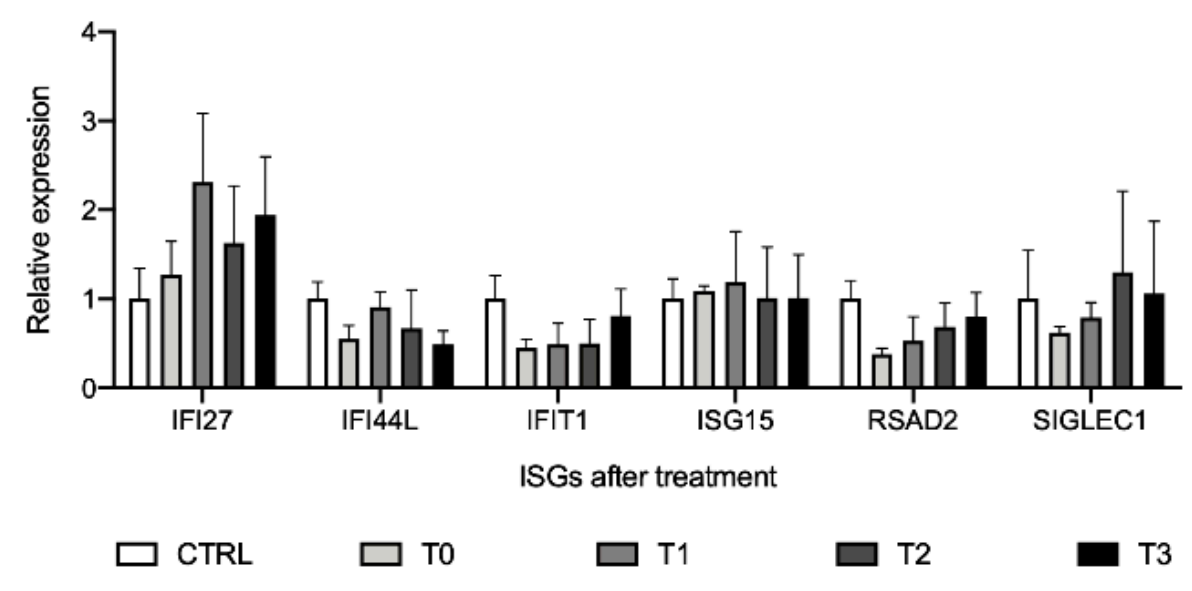

IFN signature HV2, HIGHER DOSE

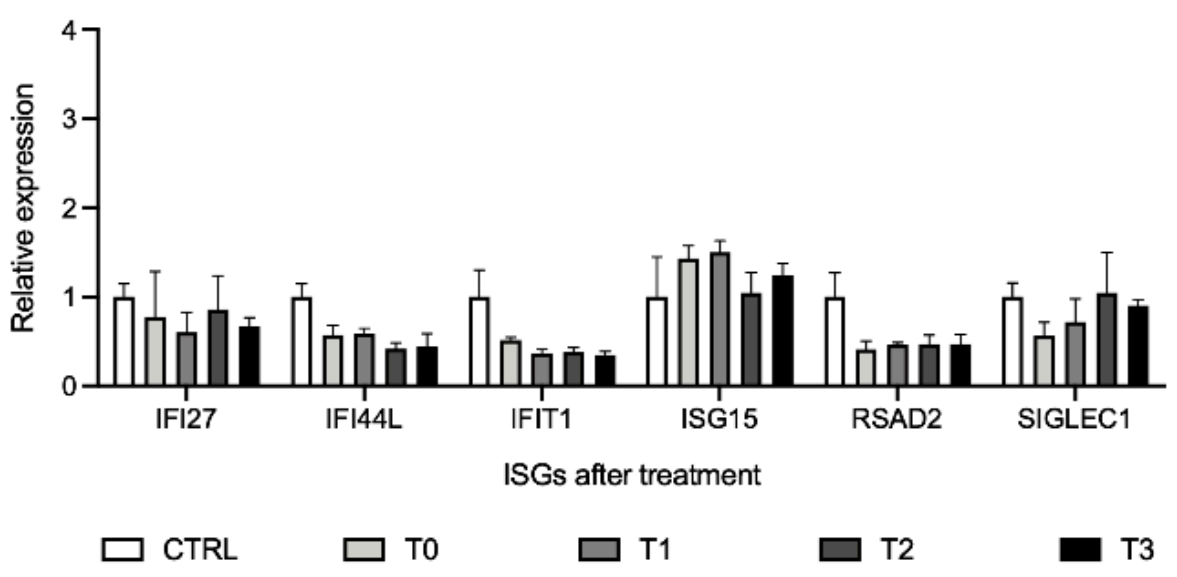

Figure 9. Interferon signature analysis in two healthy volunteers (HV1, HV2) after sulforaphane (SFN) supplement consumption at $90 \mathrm{mg}$ /day for three days, displayed as the expression levels of the six interferon-stimulated genes (ISGs). Data are shown as means and C.I. of one representative experiment and reported evaluating $2^{-\Delta \Delta C t}$ values considering a pool of healthy volunteers' cDNAs as calibrator and using HPRT1 and G6DP housekeeping genes as reference.

The IFN scores did not show significant differences in both volunteers (Tables 3 and 4).

Table 3. IFN scores calculated through the median of the relative quantifications of the six interferonstimulated genes (ISGs) of the interferon signature analysis after administration of higher-dose sulforaphane (SFN) supplement in the first healthy volunteer (HV1).

\begin{tabular}{ccccc}
\hline CTRL & T0 & T1 & T2 & T3 \\
\hline 1.00 & 0.58 & 0.85 & 0.84 & 0.91 \\
\hline
\end{tabular}

Table 4. IFN scores calculated through the median of the relative quantifications of the six interferonstimulated genes (ISGs) of the interferon signature analysis after administration of higher-dose sulforaphane (SFN) supplement in the second healthy volunteer (HV2).

\begin{tabular}{ccccc}
\hline CTRL & T0 & T1 & T2 & T3 \\
\hline 1.00 & 0.57 & 0.60 & 0.66 & 0.57 \\
\hline
\end{tabular}




\subsection{Healthy Volunteers' GSTM1 Genotype}

To evaluate GSTM1 genotype of the two healthy volunteers enrolled a genotype analysis was performed. Results indicated that the first volunteer (HV1) has a functional GSTM1 gene while the second one (HV2) presents the deletion of the gene (null genotype).

\subsection{GSTM1 Expression (HV1)}

GSTM1 expression was evaluated by RT-PCR in HV1 with the functional GSTM1 genotype before and after SFN assumption. Analyses were performed before (T0) and after SFN assumption (T1, T2, T3) comparing the GSTM1 level to no assumption condition (T0) both in lower and higher dose treatments (Figure 10).

HV1, LOWER DOSE

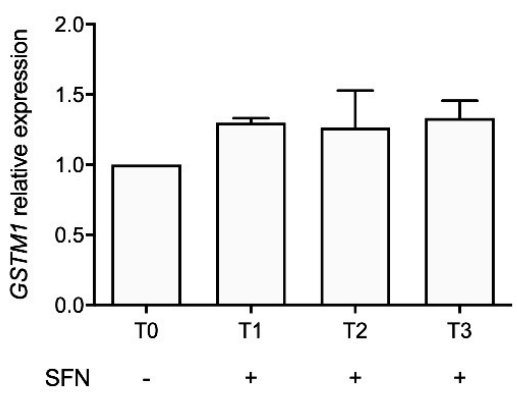

(a)
HV1, HIGHER DOSE

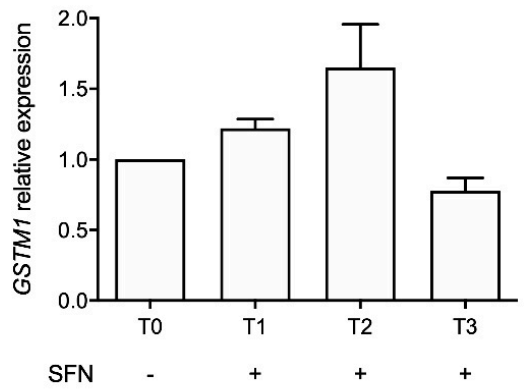

(b)

Figure 10. GSTM1 expression in the first healthy volunteer (HV1) after sulforaphane (SFN) supplement consumption at $12.6 \mathrm{mg}$ for the first day (T1), $25.2 \mathrm{mg}$ for the second and third days (T2) and $25.2 \mathrm{mg}$ for further seven days (T3) (a) and $90 \mathrm{mg} /$ day for three days (b). Data are reported as $2^{-\Delta \Delta \mathrm{Ct}}$ values considering the no assumption samples (T0) as calibrators and using GAPDH housekeeping gene as reference.

An increment trend in GSTM1 expression after SFN assumption in both treatment conditions was identified except for the last day (T3) of $90 \mathrm{mg}$ dosage.

\section{Discussion}

Autoinflammatory diseases are rare genetic conditions defined by systemic inflammatory episodes caused by innate immune system activation in the absence of pathogens, with early onset in childhood. For these conditions, treatment is focused on main disease manifestations and includes various classes of drugs such as glucocorticoids, immunomodulatory agents, antimalarials and biological drugs $[2,15,26]$. These drugs are barely effective on type I interferon generating pathway, which is constitutively active in monogenic interferonopathies like AGS, and in multifactorial interferon-related disorders like SLE, dermatomyositis, Sjögren syndrome and others [6]. In this regard, SFN, a small molecule derived from vegetables, may be useful as a possible support to dampen interferon inflammation given its ability to modulate the expression of STING [20], an upstream pathway component that mediates type I interferon production. Based on this evidence, we evaluated SFN effect in vitro in the hepatic IHH stable cell line and in vivo on two healthy volunteers.

After SFN exposure for $72 \mathrm{~h}$, a decrease in STING expression in comparison to the untreated control was detected in IHH cells. This result confirmed the ability of SFN to reduce its effects in terms of STING expression in vitro, as described by Olagnier and colleagues work in the THP-1 human monocytic cell line after SFN treatment [20]. When the IHH cells were treated with the interferon inducer cGAMP, the treatment with SFN was able to prevent the stimulation-induced increase in STING expression. Similarly, we examined the so-called interferon signature, a set of characteristic overexpressed interferonstimulated genes in patients suffering from type I interferonopathy. Interferon signature 
showed a similar behavior, demonstrating a considerably higher IFN score when cells were exposed to the cGAMP inflammatory stimulus with more than halved IFN score in cells pretreated with SFN and in presence of cGAMP. Therefore, our study provides additional information about STING expression in the presence of an inflammatory stimulus with and without pretreatment with SFN. These data provide knowledge about the role of SFN in interferon-driven inflammation suggesting a SFN preventing effect on inflammatory stimuli on IHH cell line.

Moreover, we analyzed the SFN effect on GSTM1, a gene encoding for a phase II xenobiotic detoxification enzyme, in IHH cells, previously confirmed wild type. In IHH cells we found an increased GSTM1 expression after $24 \mathrm{~h}$, while a decrease after $72 \mathrm{~h}$ using a $10 \mu \mathrm{M}$ concentration of SFN that resulted in a $30 \%$ of cell cytotoxicity. The modulation of SNF on gene expression depends on its concentration which can cause both antioxidant and pro-oxidant effects with consequent modulation on GSTM1 expression [27]. This result seems in line with the literature [27] where slightly toxic SFN concentrations can increase GSTM1 levels due to the generation of ROS that interact with NRF2 pathway which can modulate key components of inflammation-promoting gene expression contrasting the action of nuclear factor NF- $\mathrm{KB}$. Instead, after $48 \mathrm{~h}$, when ROS was significantly reduced and could not have an effect on NRF2, GSTM1 expression also decreased.

Interestingly, in the work of Yoon-Jin Lee and colleague [27] demonstrated that SFN increases the nuclear translocation of NRF2 via a ROS dependent mechanism in bronchial epithelial cells at a concentration of $10 \mu \mathrm{M}$ corresponding to around $30 \%$ of cytotoxicity: the paper describes a rapid increase in intracellular ROS levels which rapidly starts within 10 min after SFN addition, peaks at $8 \mathrm{~h}$ and gradually declines until $48 \mathrm{~h}$ [27]. From this result, it is possible to assume that as the ROS peak was found $8 \mathrm{~h}$ after treatment, and gradually decreased until $48 \mathrm{~h}$ with a consequent decrement of NRF2 nuclear accumulation, also GSTM1 induction probably could cease explaining our result of lower GSTM1 expression after $72 \mathrm{~h}$ of treatment using a slight cytotoxic dose of SFN.

As a secondary objective, we performed an exploratory analysis on STING expression levels and IFN score after SFN assumption in two healthy volunteers, to assess the possibility of extending the study by enrolling an adequate number of subjects. After preliminary data obtained in one volunteer with low SFN doses (up to $25.2 \mathrm{mg}$ /day), showing no significant effect on STING expression and IFN score, we increased the supplement dose (90 mg/day for three days), choosing a commercial supplement whose SFN content and indications resulted convenient to our purpose (Broccoraphan ${ }^{\circledR}$, Dieters). At the same time, we enrolled a second volunteer (HV2) in order to evaluate potential individual differences in response. At the same time, we performed a genotype analysis of GSTM1 since GSTM1 genotype has an important role in SFN pharmacokinetics: in particular, GSTM1 null or not functional individuals have a greater excretion of SFN and its metabolites in the first $24 \mathrm{~h}$ after SFN assumption in comparison to individuals with a functional gene [25]. Results were not consistent between the two subjects. In particular, only HV1 showed some STING expression decrease after treatment. In our experiment, HV1 was found to be wild type for GSTM1, while HV2 turned out not functional. This fact may support an interpretation of the different STING expression response to SFN in the two volunteers: the GSTM1 functional one had a more notable decrease trend in comparison to the GSTM1 non-functional individual. This result may suggest that, if SFN excretion is not rapid, as in GSTM1 functional individuals, STING repression can be possible, in a dose and time dependent manner, according to Olagnier and colleagues [20]. In fact, the lower STING level was appreciable at the last day of assumption at higher doses ( $90 \mathrm{mg} /$ day) only in the HV1 with the functional GSTM1 gene. Regarding the SFN effect on GSTM1, we can appreciate an upregulation after both treatment conditions in the HV1 except for the last day of the $90 \mathrm{mg}$ dose assumption. The induction of GSTM1 by SFN is in line with the above reported results on the IHH line where, as the initial induction of GSTM1 gradually ceases for the depletion of ROS and nuclear accumulation of NRF2, leading to the reduction of the GSTM1 expression in the last day of SFN assumption. 
The treatment with SFN did not impact the IFN score in the two volunteers. However, considering that healthy individuals did not present an inflammatory state and ISGs are downstream from STING and dependent on type I interferon production, results are reasonable, although this is a preliminary experiment. Thus, the real anti-interferon potential of SFN could be difficult to assess in unstimulated conditions.

The presence of other antioxidants could be a confounder in our analysis. However, beyond SFN, only the first supplement used (Broccoli Sprout, Love Life) also contained other antioxidants such as polyphenols. However, the much higher bioavailability of SFN in comparison to poorly bioavailable polyphenols [17], together with the data acquired using the second supplement devoid of other antioxidants, suggests that the effects highlighted in healthy donors are SFN-driven.

\section{Materials and Methods}

\subsection{Cell Culture}

The immortalized human hepatic IHH cell line was maintained in Dulbecco's modified Eagle's medium (DMEM) high glucose with the addition of 10\% fetal bovine serum (SigmaAldrich, St. Louis, MO, USA), 1.25\% L glutamine 0.2 M (EuroClone, Milan, Italy), 1\% penicillin $0.03 \mathrm{M}$ (EuroClone), streptomycin $0.02 \mathrm{M}$ (EuroClone), 1\% Hepes buffer $1 \mathrm{M}$ (EuroClone), $0.01 \%$ human insulin $10^{-4} \mathrm{M}$ (Sigma-Aldrich), and $0.04 \%$ dexamethasone $2.1 \times 10^{-3} \mathrm{M}$ (Sigma-Aldrich). Cell cultures were maintained according to standard procedures in a humidified incubator at $37^{\circ} \mathrm{C}$ and with $5 \% \mathrm{CO}_{2}$, and cell passage was performed twice a week.

\subsection{IHH Treatment}

IHH cells $\left(5 \times 10^{3}\right.$ cells/well) were exposed for $72 \mathrm{~h}$ to different concentrations of sulforaphane $(\mathrm{SFN})\left(1.25 \times 10^{-6} \mathrm{M}\right.$ to $\left.4 \times 10^{-5} \mathrm{M}\right)$ for cytotoxicity analysis evaluated by the MTT assay.

IHH cells were treated with SFN $10 \mu \mathrm{M}$ for $24 \mathrm{~h}$ and $72 \mathrm{~h}$ in presence and absence of the pro-inflammatory stimulus cGAMP (Table 5) for STING and for interferon signature analysis. In particular, cGAMP $5.9 \mu \mathrm{M}$ was added in the last 3 or $6 \mathrm{~h}$ of incubation in presence or absence of SFN $10 \mu \mathrm{M}$.

Table 5. Different combination treatment of SFN and cGAMP used on the IHH cell line.

\begin{tabular}{ccc}
\hline SFN & cGAMP & Exposure Time \\
\hline $10 \mu \mathrm{M}$ & - & $24 \mathrm{~h}$ \\
$10 \mu \mathrm{M}$ & - & $72 \mathrm{~h}$ \\
- & $5.9 \mu \mathrm{M}$ & $3 \mathrm{~h} \mathrm{cGAMP}$ \\
- & $5.9 \mu \mathrm{M}$ & $6 \mathrm{~h}$ cGAMP \\
$10 \mu \mathrm{M}$ & $5.9 \mu \mathrm{M}$ & SFN 72 h, 3 h cGAMP \\
$10 \mu \mathrm{M}$ & $5.9 \mu \mathrm{M}$ & SFN 72 h, 6 h cGAMP \\
\hline
\end{tabular}

SFN = sulforaphane; cGAMP = cyclic guanosine monophosphate-adenosine monophosphate.

\subsection{Cytotoxicity Assay}

In the last $4 \mathrm{~h}$ of treatment, a solution of 3-(4,5-dimethylthiazol-2-yl)-2,5diphenyltetrazolium (MTT) was added (final concentration $0.5 \mathrm{mg} / \mathrm{mL}$ ) and the crystals produced by metabolically active cells were solubilized with $100 \mu \mathrm{L}$ of DMSO. The absorbance was read by an Automated Microplate Reader EL 311s (Bio-Tek Instruments, Winooski, VT, USA) at 540/630 nm. Data are the means \pm SE of at least three independent experiments performed in triplicate and are reported as \% of untreated controls (absorbance treated/absorbance untreated control $\times 100)$.

\subsection{Healthy Volunteers}

Two individuals without clinical symptoms of diseases were selected for the study: both were of Caucasian ethnicity, one male and one female, more than 18 years old. 
At first, one healthy volunteer (HV1) assumed up to $25.2 \mathrm{mg} /$ day SFN (Broccoli Sprout Extract $^{\circledR}$, Love life, Cambridge, UK) for three consecutive days (T1: $24 \mathrm{~h}$ of assumption, T2: $72 \mathrm{~h}$ of assumption) and, after two weeks of stop, for other seven days (T3). Doses were decided considering the steady state concentration of SFN, which, taking $8.2 \mathrm{mg}$ every $8 \mathrm{~h}$ resulted in the $0.3-0.6 \mu \mathrm{M}$ range $[28,29]$. In particular, $4.2 \mathrm{mg}$ of SFN supplement was administered every $8 \mathrm{~h}$ for the first $24 \mathrm{~h}$ (12.6 $\mathrm{mg}$ daily), $8.2 \mathrm{mg}$ every $8 \mathrm{~h}$ for the next $48 \mathrm{~h}$ ( $25.2 \mathrm{mg}$ daily) and, after two weeks of stop, the same dosage for 7 days. Broccoli sprout extract $^{\circledR}$ Love life supplement was chosen because the amount $(2.5 \mathrm{mg}$ of SFN/capsule $600 \mathrm{mg}$ ) of SFN contained in each capsule was convenient to reach pharmacologically relevant blood concentrations of the drug. Peripheral blood samples were collected for RNA extraction before treatment (T0 no assumption), at T1, T2, and at T3 (Table 6).

Table 6. Doses and timing of sulforaphane (SFN) administrations in the first healthy volunteer enrolled (HV1).

\begin{tabular}{cc}
\hline & Lower Dose \\
\hline Dose/Day & Administration Timing \\
\hline- & No assumption (T0) \\
$12.6 \mathrm{mg}$ & Day 1 (T1) \\
$25.2 \mathrm{mg}$ & Day 2-3 (T2) \\
$25.2 \mathrm{mg}$ & 7 days assumption (T3) \\
\hline
\end{tabular}

In the second treatment, both volunteers (HV1, HV2) assumed an almost four-timeshigher dose (90 mg daily for 3 days). This time, the supplement chosen was Broccoraphan ${ }^{\circledR}$ (Dieters, Frechen, Germany) because the formulation was convenient in relation to our objectives, despite the bad taste of the supplement powder ( 15 mg SFN/g supplement$90 \mathrm{mg}$ corresponds to $6 \mathrm{~g}$ daily). Indeed, the dose should lead to an SFN steady state concentration of 1-2 $\mu \mathrm{M}[28,29]$ and was considered safe, as reported by the supplement indications. Peripheral blood samples were collected for RNA extraction before treatment (T0 no assumption), at T1, T2, and T3 for both volunteers (Table 7).

Table 7. Doses and timing of sulforaphane (SFN) administrations in both volunteers enrolled (HV1, HV2). The second-round dose treatments were considered high doses and referred as "higher dose".

\begin{tabular}{ccc}
\hline \multicolumn{3}{c}{ Higher Dose } \\
\hline Dose/Day & Administration Timing \\
\hline- & No assumption (T0) \\
$90 \mathrm{mg}$ & Day 1 (T1) \\
$90 \mathrm{mg}$ & Day 2 (T2) \\
$90 \mathrm{mg}$ & Day 3 (T3) \\
\hline
\end{tabular}

\subsection{RNA Extraction}

Total RNA of the IHH cell line was extracted with TRIzol reagent (Invitrogen, Waltham, MA, USA) while healthy volunteers' total RNA was extracted using the PAXgene Blood RNA kit (PreAnalytiX, Hombrechtikon, Switzerland) according to the manufacturer's instruction. The obtained RNA was quantified using Nanodrop 2000 spectrophotometer (ThermoFisher Scientific, Waltham, MA, USA) and reversed-transcribed into cDNA using the High-Capacity RNA-to-cDNA kit (Applied Biosystem, Waltham, MA, USA).

\subsection{Real-Time PCR}

\subsubsection{Interferon Signature and STING Analysis}

Interferon signature analysis was performed by relative quantification of six interferonstimulated genes (ISGs: IFI27, IFI44L, IFIT1, ISG15, RSAD2, SIGLEC1) by real-time PCR (RT-PCR), using UPL Probes (Roche, Basel, Switzerland), TaqMan Gene Expression Master 
Mix (Applied Biosystems), and a AB 7500 Real Time PCR system (Table 8). The same analysis was also used for STING and the housekeeping reference genes HPRT1 and G6PD.

Table 8. Universal Probe Library (UPL) probes (Roche) and primers (Eurofins Genomics) for the six interferon-stimulated genes (ISGs) of interferon signature and STING analysis by Real-Time PCR.

\begin{tabular}{|c|c|c|c|}
\hline \multicolumn{4}{|c|}{ Probes and Primers } \\
\hline GENE & $\begin{array}{c}\text { Probe } \\
(10 \mu \mathrm{M})\end{array}$ & $\begin{array}{l}\text { Primer Forward } \\
\qquad(20 \mu \mathrm{M})\end{array}$ & $\begin{array}{c}\text { Primer Reverse } \\
\qquad(20 \mu \mathrm{M})\end{array}$ \\
\hline \multicolumn{4}{|c|}{ ISGs assessed for interferon signature analysis } \\
\hline IFI27 & P. n. 21 & GTGGCCAAAGTGGTCAGG & CCAATCACAACTGTAGCAATCC \\
\hline IFI44L & P. n. 15 & TGACACTATGGGGCTAGATGG & TTGGTTTACGGGAATTAAACTGAT \\
\hline IFIT1 & P. n. 82 & TCCACAAGACAGAGAATAGCCAGAT & GCTCCAGACTATCCTTGACCTG \\
\hline ISG15 & P. n. 76 & GAGGCAGCGAACTCATCTTT & AGCATCTTCACCGTCAGGTC \\
\hline RSAD2 & P. n. 76 & ACAAATGCGGCTTCTGTTTC & GAAATGGCTCTCCACCTGAA \\
\hline SIGLEC1 & P. n. 76 & CTGCCCTGCAAGTCCTCTA & CAGCAGGTGGCTCACTGTC \\
\hline \multicolumn{4}{|c|}{ Primers and probe for measuring STING expression } \\
\hline STING & P. n. 51 & CGCCTCATTCCCTACCAG & TGCCCACAGTAACCTCCTCC \\
\hline \multicolumn{4}{|c|}{ Housekeeping genes } \\
\hline HPRT1 & P. n. 73 & TGACCTTGATTTATTTTGCATACC & CGAGCAAGACGTTCAGTCCT \\
\hline G6PD & P. n. 82 & GCAAACAGAGTGAGCCCTTC & GAGTTGCGGGCAAAGAAGT \\
\hline
\end{tabular}

The RT-PCR protocol consists of an initial denaturation for $2 \mathrm{~min}$ at $50{ }^{\circ} \mathrm{C}$ and for $95^{\circ} \mathrm{C}$ for $10 \mathrm{~min}$, followed by 40 cycles of heating at $95^{\circ} \mathrm{C}(15 \mathrm{~s})$, and then a final extension for $1 \mathrm{~min}$ at $60{ }^{\circ} \mathrm{C}$. Data were analyzed with 7500 SDS (Applied Biosystems) analysis software. Data were normalized with two housekeeping genes: G6DP and HPRT1. Relative quantification was performed using the $2^{-\Delta \Delta \mathrm{Ct}}$ method using as reference the cDNA mix of 10 healthy controls (CTRL) for ISGs while cDNA of the last day of treatment sample (T3) for STING. For interferon signature analysis, the IFN score was calculated through the median of the relative quantifications of the six ISGs.

\subsubsection{GSTM1 Expression}

RT-PCR was performed using the TaqMan ${ }^{\circledR}$ Gene Expression Assays (Hs01683722_gH, Applied Biosystems) in a Thermal Cycler Dice Real Time System (BIO-RAD). Relative quantification is represented as $2^{-\Delta \Delta C t}$ with respect to the housekeeping genes beta-actin $(A C T B)$ and glyceraldehyde 3-phosphate dehydrogenase $(G A P D H)$, setting untreated IHH and untreated donor as reference. The RT-PCR protocol for GSTM1 analysis consists of an initial denaturation for $10 \mathrm{~min}$ at $95^{\circ} \mathrm{C}$, followed by 40 cycles of heating at $95^{\circ} \mathrm{C}(15 \mathrm{~s})$ and $60{ }^{\circ} \mathrm{C}(1 \mathrm{~min})$. All experiments were carried out in duplicate and the reproducibility of the observations was confirmed in two or three independent experiments.

\subsubsection{GSTM1 Genotype}

GSTM1 genotype state was assessed by TaqMan ${ }^{\circledR}$ CNV genotyping Assays (Applied Biosystems) kit, containing forward and reverse specific primers for the interested amplicon, which, in that case, is GSTM1 and a FAM conjugated probe. Additionally, TaqMan ${ }^{\mathrm{TM}}$ Copy Number Reference Assay, human RNase P was used to assess the presence of a double copy gene. In this case, too, the kit contains forward and reverse specific primers for the interested amplicon (human RNase P) and a VIC ${ }^{\circledR}$ dye-labeled TAMRA ${ }^{\mathrm{TM}}$ probe. The thermic protocol used was $95^{\circ} \mathrm{C}$ for $10 \mathrm{~min}, 95^{\circ} \mathrm{C}$ for $15 \mathrm{sec}$, and $60{ }^{\circ} \mathrm{C}$ for $1 \mathrm{~min}$. The whole reaction was repeated for 40 cycles. Results are expressed as deleted vs. functional GSTM1.

\subsection{Statistical Analysis}

Results are presented as mean $\pm 95 \%$ confidence intervals (C.I.) from up to two independent experiments. Statistical analyses were performed using GraphPad Prism 
software (version 8.0.2). One-way ANOVA and Bonferroni's post-test were used for gene expression analysis. $p$ values $<0.05$ were considered statistically significant.

\section{Conclusions}

In conclusion, this study confirmed SFN inhibiting action on inflammatory stimuli response in vitro in terms of STING reduction and ISGs expression. We observed a STING reduction trend in vivo only in the HV1 after SFN administration. The higher efficacy of SFN in STING reduction could be related to the functional GSTM1 genotype related to a slower elimination rate and therefore a higher SFN efficacy. Since individuals were healthy and not affected by inflammatory conditions, no effect of SFN treatment was observed on ISGs expression, as already characterized by low ISGs expression. From these premises, it will be reasonable to expand the study to a larger group of healthy individuals to further investigate the association between STING modulation by SFN and GSTM1 genotype, and subsequently to evaluate these effects in patients with type I interferonopathies.

Author Contributions: Conceptualization, A.T. (Alberto Tommasini) and G.S.; data curation, E.G. and M.A.; formal analysis, E.G., M.A. and A.T. (Alessandra Tesser); funding acquisition, G.D., A.T. (Alberto Tommasini) and G.S.; investigation, E.G., M.A. and A.T. (Alessandra Tesser); methodology, E.G., M.A. and A.T. (Alessandra Tesser); project administration, E.G., M.A., A.T. (Alberto Tommasini) and G.S.; resources, G.D. and A.T. (Alberto Tommasini); software, E.G., M.A. and A.T. (Alessandra Tesser); supervision, A.T. (Alberto Tommasini) and G.S.; validation, G.D., A.T. (Alberto Tommasini) and G.S.; visualization, E.G., M.A., A.T. (Alberto Tommasini) and G.S.; writing-original draft, E.G. and M.A.; writing-review \& editing, E.G., M.A., G.D., A.T. (Alessandra Tesser) and A.T. (Alberto Tommasini). All authors have read and agreed to the published version of the manuscript.

Funding: This work was supported by Institute for Maternal and Child Health-IRCCS Burlo Garofolo RC\#24/2017 and RC\#07/2014.

Institutional Review Board Statement: The study is part of the IRCCS Burlo Garofolo project RC\#24/2017, approved by the Institutional Review Board and by the Friuli Venezia Giulia Independent Ethical Committee (2018-SPER-079-BURLO, N. 0039851, approved on 12 December 2018).

Informed Consent Statement: Written informed consent has been obtained from the participants to publish this paper.

Data Availability Statement: Raw data will be provided upon reasonable request.

Conflicts of Interest: The authors declare no conflict of interest.

\section{References}

1. Barnes, P.J. How Corticosteroids Control Inflammation: Quintiles Prize Lecture 2005. Br. J. Pharmacol. 2006, 148, 245-254. [CrossRef]

2. Diaz-Borjon, A.; Weyand, C.M.; Goronzy, J.J. Treatment of Chronic Inflammatory Diseases with Biologic Agents: Opportunities and Risks for the Elderly. Exp. Gerontol. 2006, 41, 1250-1255. [CrossRef]

3. Levin, A.D.; Wildenberg, M.E.; van den Brink, G.R. Mechanism of Action of Anti-TNF Therapy in Inflammatory Bowel Disease. J. Crohns Colitis 2016, 10, 989-997. [CrossRef]

4. Wu, C.; Wang, S.; Xian, P.; Yang, L.; Chen, Y.; Mo, X. Effect of Anti-TNF Antibodies on Clinical Response in Rheumatoid Arthritis Patients: A Meta-Analysis. Biomed Res. Int. 2016, 2016, 7185708. [CrossRef]

5. Bettiol, A.; Lopalco, G.; Emmi, G.; Cantarini, L.; Urban, M.L.; Vitale, A.; Denora, N.; Lopalco, A.; Cutrignelli, A.; Lopedota, A.; et al. Unveiling the Efficacy, Safety, and Tolerability of Anti-Interleukin-1 Treatment in Monogenic and Multifactorial Autoinflammatory Diseases. Int. J. Mol. Sci. 2019, 20, 1898. [CrossRef] [PubMed]

6. Rodero, M.P.; Crow, Y.J. Type I Interferon-Mediated Monogenic Autoinflammation: The Type I Interferonopathies, a Conceptual Overview. J. Exp. Med. 2016, 213, 2527-2538. [CrossRef]

7. Volpi, S.; Picco, P.; Caorsi, R.; Candotti, F.; Gattorno, M. Type I Interferonopathies in Pediatric Rheumatology. Pediatric Rheumatol. 2016, 14, 35. [CrossRef] [PubMed]

8. De Andrea, M.; Ravera, R.; Gioia, D.; Gariglio, M.; Landolfo, S. The Interferon System: An Overview. Eur. J. Paediatr. Neurol. 2002, 6, A41-A46. [CrossRef] [PubMed]

9. Ivashkiv, L.B.; Donlin, L.T. Regulation of Type I Interferon Responses. Nat. Rev. Immunol. 2014, 14, 36-49. [CrossRef] 
10. Rice, G.I.; Melki, I.; Frémond, M.-L.; Briggs, T.A.; Rodero, M.P.; Kitabayashi, N.; Oojageer, A.; Bader-Meunier, B.; Belot, A.; Bodemer, C.; et al. Assessment of Type I Interferon Signaling in Pediatric Inflammatory Disease. J. Clin. Immunol. 2017, 37, 123-132. [CrossRef] [PubMed]

11. Rönnblom, L.; Eloranta, M.-L. The Interferon Signature in Autoimmune Diseases. Curr. Opin. Rheumatol. 2013, 25, $248-253$. [CrossRef] [PubMed]

12. Morand, E.F.; Furie, R.; Tanaka, Y.; Bruce, I.N.; Askanase, A.D.; Richez, C.; Bae, S.-C.; Brohawn, P.Z.; Pineda, L.; Berglind, A.; et al. Trial of Anifrolumab in Active Systemic Lupus Erythematosus. N. Engl. J. Med. 2020, 382, 211-221. [CrossRef] [PubMed]

13. Moghaddas, F.; Masters, S.L. The Classification, Genetic Diagnosis and Modelling of Monogenic Autoinflammatory Disorders. Clin. Sci. 2018, 132, 1901-1924. [CrossRef] [PubMed]

14. Ferraro, R.M.; Lanzi, G.; Masneri, S.; Barisani, C.; Piovani, G.; Savio, G.; Cattalini, M.; Galli, J.; Cereda, C.; Muzi-Falconi, M.; et al. Generation of Three IPSC Lines from Fibroblasts of a Patient with Aicardi Goutières Syndrome Mutated in TREX1. Stem Cell Res. 2019, 41, 101580. [CrossRef] [PubMed]

15. Bienias, M.; Brück, N.; Griep, C.; Wolf, C.; Kretschmer, S.; Kind, B.; Tüngler, V.; Berner, R.; Lee-Kirsch, M.A. Therapeutic Approaches to Type I Interferonopathies. Curr. Rheumatol. Rep. 2018, 20, 32. [CrossRef] [PubMed]

16. Nasri, H.; Baradaran, A.; Shirzad, H.; Rafieian-Kopaei, M. New Concepts in Nutraceuticals as Alternative for Pharmaceuticals. Int. J. Prev. Med. 2014, 5, 1487-1499.

17. Houghton, C.A. Sulforaphane: Its "Coming of Age" as a Clinically Relevant Nutraceutical in the Prevention and Treatment of Chronic Disease. Available online: https:/ / www.hindawi.com/journals/omcl/2019/2716870/ (accessed on 8 April 2020).

18. Kim, J.K.; Park, S.U. Current Potential Health Benefits of Sulforaphane. EXCLI J. 2016, 15, 571-577. [CrossRef]

19. Wardyn, J.D.; Ponsford, A.H.; Sanderson, C.M. Dissecting Molecular Cross-Talk between Nrf2 and NF-KB Response Pathways. Biochem. Soc. Trans. 2015, 43, 621-626. [CrossRef] [PubMed]

20. Olagnier, D.; Brandtoft, A.M.; Gunderstofte, C.; Villadsen, N.L.; Krapp, C.; Thielke, A.L.; Laustsen, A.; Peri, S.; Hansen, A.L.; Bonefeld, L.; et al. Nrf2 Negatively Regulates STING Indicating a Link between Antiviral Sensing and Metabolic Reprogramming. Nat. Commun. 2018, 9, 3506. [CrossRef]

21. Su, X.; Jiang, X.; Meng, L.; Dong, X.; Shen, Y.; Xin, Y. Anticancer Activity of Sulforaphane: The Epigenetic Mechanisms and the Nrf2 Signaling Pathway. Available online: https://www.hindawi.com/journals/omcl/2018/5438179/ (accessed on 8 April 2020).

22. Gao, S.S.; Chen, X.Y.; Zhu, R.Z.; Choi, B.-M.; Kim, B.-R. Sulforaphane Induces Glutathione S-Transferase Isozymes Which Detoxify Aflatoxin B(1)-8,9-Epoxide in AML 12 Cells. Biofactors 2010, 36, 289-296. [CrossRef]

23. Egner, P.A.; Chen, J.G.; Wang, J.B.; Wu, Y.; Sun, Y.; Lu, J.H.; Zhu, J.; Zhang, Y.H.; Chen, Y.S.; Friesen, M.D.; et al. Bioavailability of Sulforaphane from Two Broccoli Sprout Beverages: Results of a Short Term, Cross-over Clinical Trial in Qidong, China. Cancer Prev. Res. 2011, 4, 384-395. [CrossRef] [PubMed]

24. Atwell, L.L.; Hsu, A.; Wong, C.P.; Stevens, J.F.; Bella, D.; Yu, T.-W.; Pereira, C.B.; Löhr, C.V.; Christensen, J.M.; Dashwood, R.H.; et al. Absorption and Chemopreventive Targets of Sulforaphane in Humans Following Consumption of Broccoli Sprouts or a Myrosinase-Treated Broccoli Sprout Extract. Mol. Nutr. Food Res. 2015, 59, 424-433. [CrossRef]

25. Gasper, A.V.; Al-Janobi, A.; Smith, J.A.; Bacon, J.R.; Fortun, P.; Atherton, C.; Taylor, M.A.; Hawkey, C.J.; Barrett, D.A.; Mithen, R.F. Glutathione S-Transferase M1 Polymorphism and Metabolism of Sulforaphane from Standard and High-Glucosinolate Broccoli. Am. J. Clin. Nutr. 2005, 82, 1283-1291. [CrossRef] [PubMed]

26. Harry, O.; Yasin, S.; Brunner, H. Childhood-Onset Systemic Lupus Erythematosus: A Review and Update. J. Pediatr. 2018, 196, 22-30. [CrossRef]

27. Lee, Y.-J.; Lee, S.-H. Sulforaphane Induces Antioxidative and Antiproliferative Responses by Generating Reactive Oxygen Species in Human Bronchial Epithelial BEAS-2B Cells. J. Korean Med. Sci. 2011, 26, 1474-1482. [CrossRef]

28. Vermeulen, M.; Klöpping-Ketelaars, I.W.A.A.; van den Berg, R.; Vaes, W.H.J. Bioavailability and kinetics of sulforaphane in humans after consumption of cooked versus raw broccoli. J. Agric. Food Chem. 2008, 56, 10505-10509. [CrossRef] [PubMed]

29. Houghton, C.A.; Fassett, R.G.; Coombes, J.S. Sulforaphane and Other Nutrigenomic Nrf2 Activators: Can the Clinician's Expectation Be Matched by the Reality? Oxid. Med. Cell. Longev. 2016, 2016, 7857186. [CrossRef] [PubMed] 